\title{
Hydrotherapy with using citrate buffer system in treatment of senile xerosis
}

\author{
Marina Ufimtseva ${ }^{1}$, Kseniya Sorokina ${ }^{1}$, Nadezhda Belokonova $^{2}$, Yurii Bochkarev ${ }^{1}$, and \\ Ekaterina Mylnikova ${ }^{1}$ \\ ${ }^{1}$ Ural state medical university, Department of Dermatovenereology and Life Safety, 620028, Repin \\ Str., 3, Yekaterinburg, Russian Federation \\ ${ }^{2}$ Ural state medical university, Department of General Chemistry, 620028, Repin Str., 3, \\ Yekaterinburg, Russian Federation
}

\begin{abstract}
Senile xerosis is one of the most common pathology in elderly patients. The article presents an overview of current knowledge on the subject of skin barrier function and pathophysiological mechanisms and clinical features of senile xerosis. The corneotherapeutic approach to treatment of senile xerosis is substantiated and the effectiveness of emollients for baths was justified. The $\mathrm{pH}$ of water various sources (tap and artesian water) has alkaline values, which leads to an increase in dry skin and worsening clinical symptoms of xerosis. The effectiveness of bath emollients was investigated. The analysis of the adsorption properties of bath emollients was conducted which showed that these properties depend on $\mathrm{pH}$ of water. Hydrotherapy with using the citrate buffer system increases the efficiency of treatment. 60 women, aged 75-84 years, diagnosed with senile xerosis were treated with hydrotherapy containing citrate buffer system, bath emollient (shower oil). A clinical assessment was performed at the beginning and end of the study by a dermatologist using the Overall Dry Skin Scale (ODS) and Dermatological Life Quality Index (DLQI). Hydrotherapy with using a citrate buffer system provided a significant softening of the skin, elimination of scaling, remission of pruritus and more rapid clinical effect.
\end{abstract}

\section{Introduction}

The World Health Organization (WHO) predicts that by 2050 the population of people aged over 60 years will reach two billion [1]. Ageing is a natural process associated with structural and functional changes of the organs, including the skin, as a result of the accumulated defects of intracellular structures and cells. Senile xerosis is one of the most common pathology in elderly patients which occurs in excess $50.0 \%$ among people over 65 years. The prevalence of senile xerosis increases with age [2].

Xerosis is an alteration of producing sebum and sweat, inadequate vasomotor response, amino acids and lipid deficiency, dehydration, discomfort and decreasing of quality of life [3]. The cause of senile xerosis is an age-related skin changes which are associated with intrinsic ageing of the skin [4].

The main structural change in the epidermis in senile xerosis is its moderate atrophy, which is characterized by a decrease of the number of rows of cells in each layer of the 
epidermis and a thickening of the stratum corneum. The declines in immune system function and metabolism, alteration of endocrine function, microcirculation and neurohumoral regulation in elderly subjects are observed [5].

Insufficient microcirculation is a cause of decreased keratinopoiesis, reduced synthesis of epidermal lipids, sebaceous and sweat glands atrophy which leads to impairment of the skin barrier function, transepidermal water loss (TEWL) and increased sensitivity to environmental factors, irritants, allergens and pathogens [6]. The physical barrier is represented mainly by the stratum corneum. Low water content (below $10 \mathrm{wt}$ \%) within the stratum corneum affects its plasticity and barrier function. Epidermis plays an important role in protection against stratum corneum dehydration. It depends on stratum corneum state, intercellular lipids synthesis, synthesis and transformation of filaggrin, hydrolipidic mantle state, natural humectants level [7].

The stratum corneum state plays a significant role in xerosis development. The stratum corneum structure is described as "bricks and mortar" in which corneocytes are considered to be the bricks and intercellular lipids are considered to be the mortar. Corneocytes are connected by corneodesmosomes and surrounded by intercellular lipids. Corneocytes do not have nucleus and intracellular organelles. They contain cornified envelope beneath their thick plasmalemma. The cornified envelope composed of insoluble proteins (involucrin, periplakin, envoplakin) and extracellular lipids. The proteins of the cornified envelope are connected with corneodesmosomes which forms strong intercellular bonding [4,6,7]. In elder epidermis the number of corneocytes tends to increase, however the adhesion between them decreases due to the flattering of the corneodesmosomes. It leads to impairment of the stratum corneum integrity and TEWL $[4,6]$.

Lipidogenesis activity is decreased in elderly subjects. The lipids are synthesized in the Golgi complex in keratinocytes and form multilamellar structure around corneocytes. Mature multilamellar structure is composed of ceramides (about $50.0 \%$ by weight), cholesterol $(25.0 \%)$, and free fatty acids $(25.5 \%)$. They are equal in molar ratio of about $1: 1: 1[6,7]$. In elderly epidermis the content of ceramides, cholesterol and free fatty acids is reduced which leads to impairment of the intercellular lipid membrane [6]. Moreover, the lipid processing is altered. Acid sphingomyelinase (aSMase) activity is decreased in the elderly epidermis. ASMase produces long-chain (C24) and short-chain (C16) ceramides. Activity of enzymes such as serine palmitoyltransferase (involved in the sphingosine synthesis), 3-hydroxy-3-methylglutaryl-coenzyme A reductase (involved in cholesterol synthesis), acetyl coenzyme A carboxylase (involved in fatty acids synthesis) is also decreased. It changes the ratio of lipids in the intercellular lipid membrane which leads to dehydration and xerosis development [8].

The balanced composition of humectants on the surface of corneocytes, as well as between lipid structures, allows the stratum corneum to bind water and ensure its elasticity. Humectants are water-soluble substances with a high ability to absorb water. Natural humectants include hydrolysis products of filaggrin, natural moisturizing factor (NMF), hyaluronic acid, glycerin, ceramides. Proteolysis and degradation of filaggrin products NMF components such as amino acids, sodium pyroglutamate, urea, ammonia, creatinine and other organic compounds, lactate compounds of metal ions, lactic and citric acids. T. Tezuka (1983) proved that NMF level is significantly decreased with age. Electron microscope examination showed a decrease in keratohyalin granules in senile xerosis, which would lead to reduced synthesis of profilaggrin (the precursor of filaggrin) and subsequently low filaggrin and NMF components [9].

Glycerin is a highly hygroscopic humectant and one of the important components of the epidermis water-saving system. Glycerol is a polyol with three hydroxyl groups and produced from the hydrolysis of sebum triglycerides. Transepidermal transport of glycerol is controlled by a membrane protein aquaporine 3 (AQP 3) [6,7]. Increased plasma levels of 
AQP 3 enhance the transport of the glycerol into the epidermis where it absorbs and retains water [10]. The decrease in glycerol synthesis in elderly epidermis is caused by the reduced sebum secretion up to $50.0 \%$ and/or lowered level of AQP 3. Fluhr et al. (2003) showed that decreased AQP 3 level in mice leads to skin dehydration, elasticity loss and impairment of the barrier function [11].

The growing deficiency of the level of hyaluronic acid in the epidermis and dermis, a decrease in the size of its polymers, leads not only to dehydration of the skin, but also to an impairment of the differentiation of keratinocytes and the formation of lamellar bodies [7].

All these mechanisms together lead to dehydration of the epidermis and development of senile xerosis.

Senile xerosis is characterized by scaling, decreased elasticity and turgor, small cracks. The skin takes a form of a papyrus paper and superficial blood-vessels can be seen. Clinical manifestations of senile xerosis in elderly patients are usually observed in lower extremities, lower legs in particular. However, senile xerosis can also be generalized. Senile xerosis can contribute to a pruritus, feeling of tightness of the skin, burning, tingling which negatively affects the patient's quality of life $[4,12]$. Pruritus usually appears on lower back, upper and lower extremities, and is more prominent at night, as well as after a hot bath and using detergents with aggressive surfactants [7].

Differential diagnosis of senile xerosis should include atrophoderma developing against the background of somatic diseases (cancer, myeloma, etc.) or different form of cachexia (alimentary, pituitary, cachexia strumipriva, diabetes mellitus and other pathological conditions), pseudoxanthoma elasticum, pellagra, acrodermatitis chronica atrophicans, acquired or genetically caused xeroses of the skin, accompanying other skin diseases [13].

In accordance with the pathogenetic aspects of senile xerosis, corneotherapy is the main treatment method. Dermatologists use a variety of creams and ointments, from the most common type of baby cream to specially developed lines of medical cosmetics (emollients) in order to soften and moisturize the skin with manifestations of xerosis. Using of occlusive moisturizes that mimics the effects of sebum and substances with hygroscopic components (polyglycols, polysaccharides, protein molecules, polynucleic acids) cannot provide a longterm effect because they work as long as they are on the skin. Emollients are the most effective moisturizes due to their composition. Emollients contain physiologic lipids and NMF components providing lipid replacement therapy to restore the structure of the skin barrier and prevent skin dehydration [14].

The effectiveness of emollients in senile xerosis treatment has been confirmed in various studies. S.G. Danby et al. (2016) in their study used the emollient containing urea, ceramide NP and lactate in the treatment of senile xerosis in elderly patients. The authors noted that regular use of the emollient for 28 days leads to decreased TEWL, skin pH and increased stratum corneum hydration. Moreover, there was a decrease in the activity of proteases involved in desquamation of corneocytes, an increase in the level of pyrrolidone carboxylic acid (Pyrrolidone Carboxylic Acid, PCA) and lactic acid, which are the NMF components [15].

S. Lueangarun et al. (2019) evaluated the effectiveness of an emollient containing ceramides 1, 3, 6-II. After a single application of emollient during next two hours significant increase in skin moisture was observed, which reached maximum after 4 hours. After a regular use of emollient twice daily for 28 days a significant clinical improvement in skin dryness (according to the XSS scale (R.S. Rogers $3^{\text {rd }}$ et al., 1989) was observed, a significant increase in skin moisture and a decrease in TEWL and skin $\mathrm{pH}$ were observed [16].

Many researchers studied the effectiveness of emollients with different pharmacological compositions in the treatment of xerosis and noted not only clinical improvement, a 
decrease in dryness and intensity of pruritus, but also recorded an improvement in indicators of the skin hydration using various diagnostic tools $[17,18]$.

It is recommended to use pH-neutral detergents (syndets, shower oils) for the skin cleansing. Detergents widely used in skin cleansers can emulsify lipids, which leads to TEWL and impairment of the skin barrier. It causes skin dryness, redness and swelling [19]. Soap application has a significant effect on the skin $\mathrm{pH}$ increasing $\mathrm{pH}$ by three units during 90 minutes after using and disrupting skin barrier [20]. Elderly patients diagnosed with senile xerosis should add bath oils, should not use loofah and bath sponges to avoid additional mechanical damage. It is recommended to apply emollients after bath of shower to restore skin barrier after contacting with chlorinated water which impair skin barrier [21].

In the previous studies the researchers concluded that the $\mathrm{pH}$ of water from various sources (tap, artesian) has alkaline values (from 7.1 to 8.6), increasing when heated to 1.6 units, which leads to an increase in dry skin and worsening clinical symptoms of xerosis. To reduce the $\mathrm{pH}$ of water and maintain a constant $\mathrm{pH}$ of water, regardless of temperature changes, a citrate buffer system was developed.

When adding various bath products into the water from different sources (comparable in chemical composition and properties), different adsorption capacity was discovered. Either complete absence of adsorption capacity or minimal adsorption capacity is observed [22].

\section{Materials and Methods}

The effectiveness of emollients in the treatment of senile xerosis with the inclusion of a course of hydrotherapy in 60 women, aged 75-84 years $(77.2 \pm 2.3)$, with a diagnosis of senile xerosis was studied. The inclusion criteria were: the absence of catarrhal symptoms and other signs of infectious diseases, the absence of signs of systemic inflammation according to the results of general clinical studies, the absence of chronic diseases in the stage of decompensation.

A moisturizing cream emulsion (emollient) containing distillate of sunflower oil, bioceramide, saccharides of avocado fruit (state registration certificate 77.99.1.1.U.3390.5.10 from 05.24.2010) was used. Shower oils such as substance №1 shower oil containing distillate of sunflower oil, bisabolol, almond oil (certificate of state registration 77.99.1.1. U.5812.6.06 from 06.30.2006); substance №2 - shower oil containing selectiose, thermal water, coconut oil, hydrogenated castor oil, kartama oil, evening primrose oil. Production of the citrate buffer system (patent of invention 2538084 07/25/2013 RUS) was carried out in the pharmacy network by prescription.

Patients were divided in three groups of 20 subjects: the first group comprised patients treated with hydrotherapy (the temperature of water was $37-39{ }^{\circ} \mathrm{C}$ ) containing buffer system $(\mathrm{pH}=5.5)$ and emollient (shower oil); the second comprised patients treated with hydrotherapy (the temperature of water was $37-39^{\circ} \mathrm{C}$ ) and emollient (shower oil), and the third comprised patients who did not receive hydrotherapy (control group). Hydrotherapy efficacy was assessed using an Overall Dry Skin Score (ODS) and Dermatological Life Quality Index (DLQI) [23, 24]. All subjects provided written informed consent.

The stalagmometric method was used to determine the quantitative assessment of the content and properties of surfactants in the composition of bath products (emollient). Potentiometric method was used to determine the $\mathrm{pH}$, redox potential, and buffer capacity (activated carbon was used as an adsorbent).

Production of the citrate buffer system was carried out in the pharmacy network by prescription.

Data were analyzed with "Excel 2000" and "STATISTICA-13" software. Distribution does not obey the normal distribution law. The mean, standard deviation were calculated, 
the nonparametric statistical method was used (Kruskel-Wallis test). The results were considered significant at $\mathrm{p}<0.05$.

\section{Results and Discussion}

This study showed that the adsorption properties of bath emollients depend on the type of tap water and change depending on the $\mathrm{pH}$ and the presence of a buffer system in water. Adding a citrate buffer system to tap water reduces the $\mathrm{pH}$ of water up to 5.7, which is close to the physiological $\mathrm{pH}$ of the skin.

The result of this study demonstrated that the adsorption properties of the studied shower oils improve in the presence of a citrate buffer system (Table 1.).

Table 1. The surface tension of aqueous solutions containing emollients in tap water without adding a buffer system and in the presence of a buffer system before and after adsorption.

\begin{tabular}{|c|c|c|c|c|}
\hline № & Solution & $\begin{array}{c}\sigma_{1} \text { (before } \\
\text { adsorption) }\end{array}$ & $\begin{array}{c}\sigma_{2} \text { (after } \\
\text { adsorption) }\end{array}$ & $\Delta \sigma=\sigma_{2}-\sigma_{1}$ \\
\hline \multicolumn{5}{|c|}{ Tap water, Yekaterinburg $\sigma\left(\mathrm{H}_{2} \mathrm{O}\right)=79,85 \mathrm{erg} / \mathrm{cm}^{2}$} \\
\hline 1 & Substance №1 & 60,8 & 67,9 & 7,1 \\
\hline 2 & Substance №2 & 48,5 & 53,1 & 4,6 \\
\hline \multicolumn{5}{|c|}{ Tap water + Buffer system $\sigma$ (before adsorption $)=74,5 \mathrm{erg} / \mathrm{cm}^{2}$} \\
\hline \multicolumn{5}{|c|}{$\sigma($ after adsorption $)=73,4 \mathrm{erg} / \mathrm{cm}^{2}$} \\
\hline 1 & Substance №1 & 53,7 & 68,9 & 15,2 \\
\hline 2 & Substance №2 & 49,4 & 55,0 & 5,6 \\
\hline
\end{tabular}

The highest adsorption properties were determined for shower oil - substance №1, its surface tension in tap water was $\Delta \sigma=7.1$. The surface tension more than doubled $(\Delta \sigma=$ 15.2) in the presence of a citrate buffer system. According to this result, this substance was used in a clinical study.

A course of hydrotherapy using the tap water for patients with senile xerosis was carried out in Central City Hospital №3 of Yekaterinburg. Hydrotherapy included bathing (the temperature of water was $37-39^{\circ} \mathrm{C}$ ) during 10-15 minutes №10. For the first group citrate buffer system and shower oil (substance №1) were added. Patients of the second group received shower oil (substance №1) without adding a citrate buffer system.

After bathing patients applied a moisturizer from the line of therapeutic cosmetics. All groups of patients, including controls, used a moisturizer from the line of therapeutic cosmetics twice daily.

A clinical assessment was performed at the beginning and end of the study by a dermatologist using the ODS scale. Hydrotherapy with using a citrate buffer system provided a significant softening of the skin, elimination of scaling, remission of pruritus and more rapid clinical effect than the use of a hydrotherapy with the addition of shower oil without a buffer system. On the day 10 of hydrotherapy in the first group of patients, ODS decreased from 2.8 to 0.75 , in the control group - from 2.8 to 1.95 ( $\mathrm{p}<0.01)$. Treatment efficiency in the first and second group was higher than in control group (Table 2). 
Table 2. Overall Dry Skin Scale (ODS) before and after hydrotherapy in patients with senile xerosis.

\begin{tabular}{|c|c|c|c|c|c|}
\hline \multicolumn{2}{|c|}{$\begin{array}{c}\text { I group } \\
\text { Buffer system+shower } \\
\text { oil }\end{array}$} & \multicolumn{2}{c|}{$\begin{array}{c}\text { II group } \\
\text { Shower oil }\end{array}$} & \multicolumn{2}{c|}{ III control group } \\
\hline $\begin{array}{c}\text { Before } \\
\text { treatment }\end{array}$ & $\begin{array}{c}\text { After } \\
\text { treatment }\end{array}$ & $\begin{array}{c}\text { Before } \\
\text { treatment }\end{array}$ & $\begin{array}{c}\text { After } \\
\text { treatment }\end{array}$ & $\begin{array}{c}\text { Before } \\
\text { treatment }\end{array}$ & $\begin{array}{c}\text { After } \\
\text { treatment }\end{array}$ \\
\hline 2.8 & 0.75 & 2.85 & 1.4 & 2.8 & 1.95 \\
\hline
\end{tabular}

Hydrotherapy treatment of senile xerosis resolved clinical symptoms more rapidly which increased patients' quality of life. DLQI index decreased from 9.25 to 0.3 and from 8.8 to $4.0(\mathrm{p}<0,01)$ for the first and the second group respectively. In patients of the control group who received emollient without hydrotherapy, the quality of life index decreased more slowly - from 8.55 to 5.1 .

\section{Conclusion}

In this study we found, that citrate buffer system reduces $\mathrm{pH}$ of tap water up to 5,7 which is physiological for skin. Moreover, the citrate buffer system improves adsorption properties of bathing emollients. Hydrotherapy with using the citrate buffer system increases the efficiency of treatment and can be recommended at the outpatient stage of dermatovenereological care.

\section{References}

1. Global strategy and action plan on ageing and health. (Geneva: World Health Organization, 2017)

2. R. Reszke, D. Pełka, A. Walasek, Z. Machaj, A. Reich, Int. J. Dermatol., 54, e332-338 (2015)

3. E. Ernandes, A. Margolina, A. Petruhina, Lipid barrier of the skin and cosmetics (OOO "Firma KLAVEL", 2005)

4. N. Katoh, D. Tennstedt, G. Abellan van Kan, M. Saint Aroman, A. Loir, D. Bacqueville, L. Duprat, B. Guiraud, S. Bessou-Touya, H. Duplan, JEADV, 32, 1-20 (2018)

5. T.G. Berger, M. Steinhoff, Semin. Cutan. Med. Surg., 30, 113-117 (2011)

6. E.H. Choi, Clin. Dermatol., 37, 336-347 (2019)

7. O.B. Tamrazova, Russian Journal of Clinical Dermatology and Venereology, 18, 193$202(2019)$

8. J-M. Jensen, M. Förl, S. Winoto-Morbach, S. Seite, M. Schunck, E. Proksch, S. Schütze, Exp. Dermatol., 14, 609-618 (2005)

9. M. Takahashi, T. Tezuka, Dermatologica, 166, 57-61 (1983)

10. H. Kiwada, J.M. Barichello, N. Jamakawa, Int. J. Pharm., 357, 199-205 (2008)

11. J.W. Fluhr, M. Mao-Qiang, B.E. Brown, P.W. Wertz, D. Crumrine, J.P. Sundberg, K.R. Feingold, P.M. Elias, J. Invest. Dermatol., 120, 728-737 (2003)

12. S. Lawton, Nurs. Older People, 30, 42-48 (2018)

13. O.M. Burylina, A.V. Karpova, Cosmetology: a clinical guide (GEOTAR-Media, 2018)

14. E.I. Ernandes, Dry skin. "My speciality is cosmetology” series (OOO "ID "Kosmetika i medicina", 2012)

15. S.G. Danby, K. Brown, T. Higgs-Bayliss, J. Chittock, L. Albenali, M.J. Corka, Skin Pharmacol. Physiol., 29, 135-147 (2016) 
16. S. Lueangarun, P. Tragulplaingam, S. Sugkraroek, T. Tempark, Dermatol, Ther., 32, e13090 (2019)

17. K. Hayama, Y. Takano, J. Tamura, H. Tagami, T. Terui, Australas J. Dermatol., 56, 36-39 (2015)

18. R. Izumi, O. Negi, A. Kamo, Y. Suga, S. Matsukuma, K. Takamori, J. Cosmet. Dermatol., 16, e37-e41 (2017)

19. H. Törmä, M. Lindberg, B. Berne, J. Invest. Dermatol., 128, 1212-1219 (2008)

20. H. Mücke, K.T. Mohr, A. Rümmler, P. Wutzler, Pharmazie, 48, 468-469 (1993)

21. M.R. Perkin, J. Craven, K. Logan, D. Strachan, T. Marrs, S. Radulovic, L.E. Campbell, S.F. MacCallum, W.H.I. McLean, G. Lack, C. Flohr, J. Allergy Clin. Immunol., 138, 509-516 (2016)

22. K.N. Sorokina, M.A. Ufimtseva, N.A. Belokonova, N.A. Naronova, Russian Journal of Clinical Dermatology and Venereology, 12, 75-79 (2014)

23. J. Serup, Skin Res. Technol., 1, 109-114 (1995)

24. A.Y. Finlay, G.K. Khan, Clin. Exp. Dermatol., 19, 210-216 (1994) 